\title{
LA VARIABLE DE DISCAPACIDAD EN LA POLÍTICA CULTURAL EN CHILE: BALANCES Y DESAFÍOS ${ }^{1}$
}

\section{Paula Milenka Cancino González², Alejandra Karina Reinoso Aguiló ${ }^{3}$ y Constanza Edith} Rojas Rodríguez ${ }^{4}$

\section{Resumen}

Introducción: El origen de la discapacidad no se encuentra únicamente en el individuo, sino que en la interacción negativa entre factores individuales y contextuales. Estos últimos determinan barreras u oportunidades de participación y al variar, pueden modificar la condición de discapacidad e incluso eliminarla aún cuando el individuo mantenga su diagnóstico de salud. De aquí la vinculación y la importancia de las acciones de la política pública en torno a la temática. Esta investigación, pondrá su foco en la Política Cultural de Chile la cual establece entre sus principios la promoción del acceso y la participación de la comunidad en iniciativas artístico-culturales, lo que sin duda incluye la variable discapacidad.

Objetivo:El objetivo de la investigación es contribuir a la vinculación efectiva y apropiada entre la variable discapacidad y el acceso a la cultura desde las políticas públicas chilenas, explorando desafíos para la gestión cultural en esta materia.

Metodología: Basándose en el concepto de accesibilidad universal, y con una metodología de tipo cualitativa, se analizan los programas de política pública asociados a la variable discapacidad de tres servicios del Estado: Dirección de Bibliotecas Archivos y Museos, el Servicio Nacional de Discapacidad y el Consejo Nacional de la Cultura y las Artes.

Resultados:Se evidencia luego de realizados los análisis, que si bien desde el Estado chileno se integran líneas que abordan temas de discapacidad y cultura, lo que sin duda debe ser reconocido como un avance, estas referencias no garantizan de manera óptima la accesibilidad universal.

Conclusion: El gestor cultural aparece como un actor relevante en la formulación de la política cultural y por tanto en la construcción de la situación de discapacidad.

\section{Palabras Clave}

Personas con discapacidad, Cultura, Políticas públicas.

\footnotetext{
Ponencia presentada al $2^{\circ}$ Congreso Nacional de Gestión Cultural, Participación ciudadana, comunidad e incidencia en la gestión cultural, realizado en Santiago de Chile, los días 24, 25 y 26 de Noviembre de 2016.

Terapeuta Ocupacional, Licenciada en Ciencias de la Ocupación, Universidad de Chile, Diplomada en Gestión Cultural, Pontificia Universidad Católica de Chile. Diplomada en "Inclusión Social, Derechos Humanos y Discapacidad", Universidad de Santiago e Instituto Nacional de Derechos Humanos. Número de ResearcherID:G-3994-2017 ORCID ID:0000-0002-8010-3151. Correo postal: Padre Miguel de Olivares 1444, depto 1006, Santiago, Chile. Correo electrónico: paulamilenka@gmail.com. Teléfono: 979578972.

Periodista, Licenciada en Comunicación Social, Pontificia Universidad Católica de Chile. Diplomado en Gestión Cultural. Pontificia Universidad Católica de Chile. N ResearcherID: G-2516-2017 ORCID ID 0000-0002-1257-8817 Correo postal: Pasaje Palguín 5538, Macul, Santiago, Chile. Correo electrónico: reinosoaguilo@gmail.com teléfono: 56995130778

4 Administrador Público, Licenciada en Ciencias Políticas y Gubernamentales con mención en Ciencia Política, Universidad de Chile. Diplomada en Gestión Cultural, Pontificia Universidad Católica de Chile. Número ResearcherID: G-3919-2017 ORCID ID: 0000-0002-3707-1799 Correo Postal: Alfredo Barros Errázuriz 1925, Depto. 501, Providencia, Santiago, Chile. Correo electrónico: conyrojas82@gmail.com. Teléfono: 569 42170534
} 


\section{Abstract}

Introduction: The origin of the disability is not only in the individual. It is on the interaction between individual and contextual factors and these last ones determine opportunities of participation.

When factors are varying, they can modify the condition of disability and eliminate it, even if the person maintains his diagnosis of health. In this sense, public policies are important to address the issue.

This research will focus on the Chilean Cultural Policy, which establishes among its principles: the promotion of access and participation of the community in artistic and cultural initiatives. It is included the variable disability, undoubtedly.

Objetive: The objective of the research is to contribute to the effective and appropriate link between the variables "disability" and "acces to culture" from public policies, exploring cultural management challenges.

Methodology: Based on the concept of universal accessibility, this research has a Methodology of qualitative type. It analyses the public policy programs associated with the variable disability of three State services: Directorate of Libraries, Archives and Museums, National Disability Service and National Council of Culture and Arts.

Results:It is evident from the analysis that references of disability and culture are integrated by the Chilean State, and it should certainly be recognized as an advance, but these references do not guarantee the optimum universal accessibility.

Conclusions: The cultural manager appears as a relevant actor in the formulation of cultural policy and therefore in the construction of the disability situation.
\end{abstract}

\title{
Keywords
}

Persons with disabilities, Culture, Public Policies

Fecha recepción: 30/03/2017

Fecha aceptación: 20/06/2017

\section{INTRODUCCIÓN}

La Política Cultural de Chile 2011-2016 señala entre sus principios la participación y destaca como objetivo, la "promoción del acceso y la participación de la comunidad en iniciativas artístico-culturales" (Consejo Nacional de la Cultura y las Artes, 2011: 60). En el marco del acceso este objetivo alude, aunque de manera implícita, a la variable discapacidad. Asimismo, el Servicio Nacional de la Discapacidad (SENADIS), señala en sus líneas de acción: promover la participación de las personas en situación de discapacidad en las actividades culturales de las artes, a través del mejoramiento de la accesibilidad y de una oferta programática más amplia y de carácte inclusivo (Servicio Nacional de la Discapacidad, 2016)

A la luz de estos lineamientos de Política Pública, est investigación busca esclarecer y evaluar la vinculación real que ha existido entre las políticas asociadas al ámbito cultural y la variable discapacidad, a través del análisis de programas de política, con una metodología cualitativa de estudio de caso. Se reflexionará sobre: ¿cómo se ha considerado la variable de discapacidad desde las política públicas culturales? La manera en que se considera la variable discapacidad en las políticas públicas culturales ¿Contribuye o no a disminuir las barreras sociales? Y, a partir de ello, ¿cómo podemos aportar, en tanto gestores culturales, a generar un cambio en la comprensión de la variable discapacidad en el contexto cultural chileno?

El objetivo de esta investigación es contribuir a la vinculación efectiva y apropiada entre la variable discapacidad y el acceso a la cultura desde las políticas públicas chilenas, explorando desafíos para la gestión cultural en esta materia, a través de la comprensión de la forma en que se ha considerado la situación de dis capacidad en los programas e instrumentos de política pública, evidenciando si esta incorporación ha contribuido a eliminar las limitaciones de acceso a la cultura, y finalmente definir cuáles son los principales desafíos en esta materia.

\section{MARCO TEÓRICO Y CONCEPTUAL}

El concepto de discapacidad se entiende como el resultado de una compleja relación entre la condición de salud de una persona, sus factores personales, y los factores externos que representan las circunstancias en 
las que vive esa persona" (Organización Mundial de la Salud, 2001: 27). Según lo aportado por la Convención Internacional de los Derechos de las Personas en situación de Discapacidad y su protocolo facultativo en su preámbulo, "la discapacidad es un concepto que evoluciona y que resulta de la interacción entre las personas con deficiencias y las barreras debidas a la actitud y al entorno que evitan su participación plena y efectiva en la sociedad, en igualdad de condiciones con las demás" (Organización de las Naciones Unidas, 2006: 2).

Lo anterior indica que la discapacidad no tiene su origen únicamente en el individuo. Si los factores que la producen varían, esta puede o no existir, incluso si el individuo mantiene su diagnóstico de salud. De aquí la vinculación y la importancia de las acciones de la política pública en torno a la temática. Las políticas públicas suponen, escoger "qué objetivos se alcanzarán, qué se hará y qué no se hará, a quienes se privilegiará en la discusión política, qué instrumentos o mecanismos se utilizarán para escoger las alternativas, y cómo se enfrentarán las consecuencias de la política, entre otros aspectos" (Kay, 2006: 2). Si se piensa en las políticas públicas culturales estas implican soluciones que se despliegan a raíz de decisiones, y por tanto son "la suma de actividades gubernamentales, actuando de manera directa o a través de agentes destinadas a tener influencia sobre la vida de los ciudadanos" (Peters, 2013: 4).

La cultura se constituye como un derecho y una necesidad humana. Por esta razón, en las políticas culturales se integra, ya sea explícita o implícitamente, el concepto de la accesibilidad universal. Este concepto asociado a la participación de todas las personas, está íntimamente ligado con la situación de discapacidad, en cuanto esta condición implica limitantes para el acceso a ciertas expresiones culturales. La accesibilidad universal, no es la única perspectiva con la que se ha atendido el concepto de discapacidad en materia cultural, también se vincula como herramienta para tratar las condiciones de salud como por ejemplo el arte-terapia; para fines de este estudio se considerará este enfoque en cuanto trabaja con los factores externos y contextuales de la situación de discapacidad.

Alrededor del mundo, la accesibilidad universal y el concepto de discapacidad ha sido materia de preocupación para las políticas culturales. Ejemplo de ello es lo estipulado en el modelo público español que, través de la Estrategia integral española de cultura para todos, integra el concepto de accesibilidad universal y se explica que "consiste en intervenir en la forma en que funciona la organización social y en el medio físico en el que ésta se asienta con el fin de suprimir los obstáculos a los que se enfrentan las personas con discapacidad y que les dificultan o impiden el ejercicio de sus derechos ciudadanos"(Ministerio de Cultura y Ministerio de Sanidad, Política Social e Igualdad de España, 2011: 1).

La Estrategia integral española cultura para todos incluye entre sus objetivos: (1) garantizar la plena accesibilidad universal y el diseño para todos en los espacios, acciones y servicios culturales, tanto temporales como permanentes, que gestione el Ministerio de Cultura y sus organismos autónomos; (2) promover el acceso de las personas con discapacidad a los fondos bibliográficos y documentales, facilitando los recursos de apoyo necesarios; (3) fomentar la participación activa de las personas con discapacidad en la creación artística en los diferentes ámbitos de ésta; (4) promover la investigación, desarrollo e innovación en tecnologías que faciliten hacer accesibles los diferentes contenidos culturales y los dispositivos y procesos de acceso a los mismos; (5) fomentar en los espacios y acciones culturales la contratación de personas con discapacidad; (6) promover una política de obras y compras públicas en los espacios y acciones culturales orientada a garantizar la accesibilidad a través del diseño para todos, la responsabilidad social empresarial y el empleo de las personas con discapacidad; (7) promover la formación en discapacidad y accesibilidad de los profesionales de la cultura y del personal de los espacios culturales para que puedan identificar y dar respuesta a las necesidades de las personas con discapacidad; (8) velar por el mantenimiento del régimen especial de la normativa sobre propiedad intelectual que favorece el acceso de las personas con discapacidad a los materiales culturales; (9) realizar campañas informativas y de divulgación cultural, en formatos accesibles, para dar a conocer y fomentar la asistencia y participación de las personas con discapacidad en las ofertas culturales; (10) garantizar la participación real y efectiva de los propios usuarios y de sus organizaciones representativas en la ejecución y seguimiento de esta Estrategia.

Estos objetivos operacionalizan de manera clara los propósitos de la política cultural en materia de discapacidad y constituyen un buen ejemplo desde un punto de vista del acceso, donde el Estado se hace cargo de los factores que inciden en la situación de discapacidad y pone a disposición de los ciudadanos las herramientas para entregar capacidad de acceso a bienes y servicios culturales.

En Chile existen instrumentos legales y entidades públicas involucradas directamente en la problemática que se analiza. En primer lugar, dentro de los recursos 
legales se encuentran la Ley 20.422 que establece Normas sobre Igualdad de Oportunidades e Inclusión Social de Personas con Discapacidad y la Ley 17.336 de Propiedad Intelectual.

Ambas contienen artículos que vinculan directamente las variables discapacidad y cultura, sin embargo poseen ciertas ambigüedades: no establecen reglamentos ni fichas técnicas para efectuar las adecuaciones para personas con discapacidad, y si bien la ley 20.422 cumple con los principios de la Declaración de Derechos Humanos y la Convención Internacional de Derechos de las Personas con Discapacidad, no genera la totalidad de normas que se requieren para garantizar el acceso a la cultura.

Paralelamente, en el Congreso Nacional, continúan en trámite seis proyectos de Ley destinados a disminuir las barreras de la discapacidad en la cultura. Uno de ellos, ingresado en 2008, que busca establecer requisitos para la inclusión en conciertos y eventos musicales que se presentan en el país. Otros, en cambio, están enfocado en uno de los medios de comunicación con más alcance de Chile, como lo es la televisión. Un proyecto ingresado hace dos años persigue incluir el establecimiento de la lengua de señas en los programas con contenido musical y espectáculos en vivo transmitidos por la TV. También se busca incorporar este sistema y el subtitulado oculto en programas culturales e infantiles. Además, entre las buenas intenciones que siguen en trámite en el Pode Legislativo, hace ya más de un año se persigue garanti zar el acceso de las personas ciegas, con discapacidad visual o con otras dificultades, a las obras publicadas en texto impreso.

Asimismo, existen dos entidades públicas que tienen responsabilidad directa en la temática, ambas con un programa de acción basado en principios, líneas estratégicas y objetivos a cumplir. Sus labores son el resultado de una planificación que considera las variables discapacidad y cultura velando, como instituciones de Estado, garantizar el resguardo de los derechos humanos a todas las personas. Por un lado, está el Servicio Nacional de Discapacidad (SENADIS), servicio público dependiente del Ministerio de Desarrollo Social, el cua busca "promover en los servicios públicos relacionados con la cultura y las artes, la incorporación de la variable de discapacidad en sus políticas y oferta programática" (Servicio Nacional de la Discapacidad, s.f) con el objetivo de "mejorar la calidad de vida de las personas en situación de discapacidad por medio de la participación activa en las actividades culturales y de las artes". Otra forma de promover la participación activa en cultura de las personas en situación de discapacidad ha sido a través del Fondo Nacional de Proyectos Inclusivos, el cual en 2015 destinó \$266.000 millones a 31 proyectos seleccionados en la línea de cultura, en una clara apelación al principio de subsidiariedad y entendiendo el arte cas exclusivamente como herramienta de rehabilitación o difusión de los derechos de las personas con discapacidad. Finalmente, SENADIS, engloba la cultura junto con tiempo libre, deporte y recreación, siendo mínimamente específica en cuanto al arte y todas sus áreas.

Por otro lado, el Consejo Nacional de la Cultura y las Artes (CNCA), dependiente del Ministerio de Educación, asume en su misión promover un desarrollo cultural armónico, pluralista y equitativo entre los habitantes del país, a través del fomento y difusión de la creación artística nacional; así como de la preservación, promoción y difusión del patrimonio cultural chileno, adoptando iniciativas públicas que estimulen una participación activa de la ciudadanía en el logro de tales fines (Consejo Nacional de la Cultura y las Artes, 2016). Sin embargo, no dedica ninguna mención directa a la discapacidad en su Política Cultural 2011-2016, ni en su Cuenta Pública Participativa 2016

Si bien parecería coherente establecer una coordinación interinstitucional entre ambos servicios, solo en el año 2015 iniciaron un trabajo conjunto entre Senadis y el Consejo Nacional de la Cultura y las Artes, además de participar activamente en la mesa del Plan Nacional de a Lectura. Esta eventualidad denota la poca comunicación entre ambas entidades, lo que expone acciones y programas miopes, en donde el acceso a la cultura para personas con discapacidad es una temática de segunda categoría.

\section{MÉTODO}

Los conceptos desarrollados en la sección anterior serán la base para las dimensiones de análisis en relación a los programas de política cultural y su vinculación con la discapacidad implementados en Chile. Las dimensiones que se analizarán son: (1) Entrega de garantías para e acceso a espacios culturales, (2) Facilitación de recursos de apoyo en relación a bienes y servicios culturales; (3) Fomento de la participación activa de las personas con discapacidad en la creación artística; (4) Fomento de la contratación de personas con discapacidad en los espacios y acciones culturales; (5) Realización de campañas informativas sobre el programa en formatos accesibles; (6) Promoción de la formación en discapacidad y accesibilidad de los profesionales de la cultura; (7) Promoción de 
la investigación, desarrollo e innovación en tecnologías que faciliten el acceso.

Para efectos de la presente investigación, se utilizará una metodología cualitativa que permite a partir del análisis detallado de los fenómenos, generar nuevas categorías de análisis acorde a la realidad que se está observando. El estudio será descriptivo en cuanto el interés se centra en la sistematización de información, y busca determinar propiedades, características y desafíos para la política cultural. Además de dar cuenta del estado de las cosas, interpreta un estado actual en torno a un estado deseado. En este ámbito de acción el presente estudio, es asimismo de tipo exploratorio, se propone observar los desafíos y entregar recomendaciones hacia una estrategia coordinada de acción en la materia.

La política cultural de Chile se ha vinculado con los temas de discapacidad desde los programas que emanan de cada uno de los servicios asociados a las materias de cultura e inclusión. Estos programas serán los sujetos de investigación. Para el análisis se considerarán el Consejo Nacional de la Cultura y las Artes, el Servicio Nacional de Discapacidad y la Dirección de Bibliotecas, Archivos y Museos, del Ministerio de Educación.

El Consejo Nacional de la Cultura y las Artes, si bien cuenta con programas a los cuales podría acceder la población en situación de discapacidad para mejorar su participación tanto en calidad de público, artista o gestor, por ejemplo a través de los fondos de cultura, dispone solo de tres programas o fondos de cultura con líneas exclusivas o que se orientan explícitamente a generar/ mejorar condiciones de inclusión para el acceso a la cultura en quienes están en situación de discapacidad. Una de estas convocatorias es el Programa de Fomento del Arte en la Educación, ACCIONA, que en el año 2015 abrió una convocatoria exclusiva para artistas en situación de discapacidad y los invitó a ser parte del Programa, para promover una participación inclusiva del Arte en la Educación. Las otras dos líneas, son parte del Fondo del Libro y la Lectura y la apertura de una nueva modalidad en el Fondo Audiovisual dedicado a la población en situación de discapacidad. Estas últimas fueron abiertas para proyectos a realizar el año 2017, por lo que aún no se conoce cuál ha sido su desarrollo.

El Servicio Nacional de Discapacidad (SENADIS) se encarga de velar por la inclusión de la población en situación de discapacidad para visibilizar y abordar de manera multisectorial sus diversas dimensiones y necesidades. El Fondo Nacional de Proyectos Inclusivos, dependiente de este servicio, financia proyectos en ocho áreas diferentes, y una corresponde a cultura.
Finalmente, la Dirección de Bibliotecas, Archivos y Museos (DIBAM) no cuenta con programas permanentes que se dediquen exclusivamente a avanzar en materia de inclusión, pero sí ha implementado iniciativas dentro de sus servicios, como son el mejoramiento de bibliotecas públicas para acceder a espacios culturales (caso de Biblioteca Santiago que obtiene premio de Sello Inclusivo 2014 en categoría de espacios públicos); mejoramiento de TICS que permitan un acceso inclusivo a la cultura (Biblioteca Nacional presenta portal cultural para ciegos); como también de acceso en espacios patrimoniales (Convenio DIBAM-fundación AMOMA para generar proyectos de inclusión a población con discapacidad sensorial en museos).

Para evaluar la efectividad de la política a través de los programas analizados, se levantará evidencia desde los documentos públicos asociados a los programas (bases de postulación, resoluciones, comunicados del servicio) para cada una de las dimensiones estipuladas. Resulta interesante constatar que la mayoría de los programas que existen hoy en la institucionalidad son iniciativas creadas durante los últimos dos años, estando muchas de ellas aún en una etapa de desarrollo. Es por esto que solo se han evaluado los programas en términos de diseño y no de implementación. Para la sistematización de esta información se ha creado una matriz especialmente para estos fines donde se organiza la evidencia.

\section{Resultados}

La primera dimensión es la entrega de garantías para el acceso a espacios culturales, específicamente lo que tiene que ver con infraestructura cultural. De los programas analizados, solo el Fondo del Libro y la Lectura incluye una modalidad destinada al mejoramiento de espacios culturales convencionales o no convencionales para la lectura, y en particular para el mejoramiento de infraestructura en bibliotecas para personas en situación de discapacidad. Asimismo, el programa de inclusión de la Biblioteca Santiago, habilita espacios e infraestructura para el acceso a personas con movilidad reducida. Por lo tanto, las acciones para garantizar el acceso a espacios culturales, desde el Estado, se han visto principalmente asociadas a iniciativas vinculadas al área de fomento de la lectura.

La facilitación de recursos de apoyo en torno a bienes y servicios culturales, es una de las dimensiones que más se ha abordado desde el Estado. Se han destinado 
recursos para la mejora de servicios, incorporación de elementos tecnológicos para interpretar los guione museográficos, y revisión del sitio web de DIBAM en conjunto con AMOMA para acceder a material audiovisua y de lectura, por ejemplo. Sin embargo, en su mayoría a entrega de recursos son administrados por privados que llevan a cabo sus iniciativas culturales.

El fomento a la participación activa de las personas en situación de discapacidad en la creación artística está cubierta principalmente por el Programa de Fomento del Arte en la Educación, ACCIONA, que realiza una convocatoria exclusiva para artistas en situación de discapacidad. Sin embargo, esta iniciativa si bien e vincula con el fomento a la creación y participación en el arte, tiene como fin aportar al mejoramiento de la calidad de la educación a través del desarrollo de la creatividad, la formación en artes y cultura, y el desarrollo de capacidades socio afectivas de estudiantes pertenecientes a establecimientos educacionales municipales y subvencionados. Por lo tanto, tiene una perspectiva íntegramente educativa. Sobre los otros programas, el fondo de SENADIS, permite una apertura en este sentido y eventualmente podría financiar proyectos vinculados a la creación por parte de personas en situación de discapacidad, sin embargo, no existe un programa que se encargue de este tema de manera específica.

El fomento a la contratación de personas en situación de discapacidad en los espacios y acciones culturales, solo se ve garantizado por el Fondo Audiovisual 2017 de distribución con fin de integración de audiencias de personas en situación de discapacidad, ya que la evaluación incluye un incentivo a que participen personas en situación de discapacidad dentro del equipo de trabajo. Las demás iniciativas, si bien no poseen garantías de este tipo, sí entregan apertura en este sentido, por ejemplo el Convenio DIBAM-AMOMA, como proyecto piloto podría generar contrataciones de personas en situación de discapacidad en materia de lengua de señas. En el acuerdo, DIBAM se compromete a coordinar una mesa o reuniones en las que representantes de organizaciones de personas con diversos orígenes y tipos de discapacidad puedan aportar experiencias sobre la construcción colaborativa de guiones museográficos inclusivos.

Si los programas analizados incluyen la variable discapacidad, deben poseer campañas informativas sobre el programa en formatos accesibles. Sin embargo, solo en uno de los programas analizados se evidenció una campaña que incluía lengua de señas en sus canales de difusión. Esta dimensión también se relaciona con la promoción de la formación en temáticas de discapaci- dad y accesibilidad a los profesionales de la cultura. En este sentido, el acuerdo DIBAM-AMOMA considera la realización de capacitaciones para los funcionarios de DIBAM en lengua de señas. Asimismo, a los funcionarios de la Biblioteca de Santiago, se les han entregado capacitaciones en lengua de señas para la atención a público. Es interesante además constatar que el Programa de Fomento del Arte en la Educación, ACCIONA, incentiva a que artistas que estén en situación de discapacidad trabajen conjuntamente con docentes en escuelas, lo cual permite la integración de diferentes formaciones y perspectivas en los proyectos.

Finalmente, sobre la promoción en investigación desarrollo e innovación en tecnologías que faciliten el acceso para personas en situación de discapacidad, estas se ven incluidas en iniciativas vinculadas al audiovisual. Se promueve la incorporación de formatos inclusivos en pos de mejorar el acceso a obras audiovisuales para personas en situación de discapacidad, y en fomento a la lectura, específicamente en el uso de las TICS para mejorar el acceso a información educativa y cultural disponible en la Biblioteca Nacional.

Si observamos de manera conjunta las dimensiones de análisis, podemos concluir que hay algunos elementos en que la política pública ha avanzado un poco más que en otros. Sin embargo, es importante hacer notar que de los programas analizados son en su mayoría fondos concursables, siendo también los que poseen mayo alcance de recursos y están directamente vinculados a la política cultural. El Estado replica su condición de subsidiariedad en este tema. Cabe hacer notar en este sentido, que hay una carencia de iniciativas que sean pensadas de manera permanente y que se enfoquen a problemáticas particulares. Asimismo, el tema cultural sobre todo cuando está mirado desde la discapacidad se integra con otras dimensiones como el deporte y la inclusión laboral, pero no se le entrega una mirada particular a las dinámicas que posee el sector.

Los sectores artísticos también están representados de manera desigual en los programas. Encontramos iniciativas en las áreas de fomento a la Lectura y Audiovisual principalmente. Sin embargo, no hay programas específicos que aborden las problemáticas asociadas a las artes escénicas y a la música, por ejemplo, así como tampoco a poner en valor el rescate patrimonial. Todo esto sin perjuicio de que algunas iniciativas, como el Fondo Nacional de Proyectos Inclusivos, deje abierta la posibilidad de incluir proyectos de cualquier naturaleza.

Estos elementos son interesantes de revelar, ya que si bien desde el Estado chileno se integran líneas que 
abordan temas de discapacidad y cultura, lo que sin duda debe ser reconocido como un avance, estas referencias no garantizan, de manera óptima, la accesibilidad universal.

\section{DISCUSIÓN}

El gestor cultural aparece como un actor relevante en la formulación de la política cultural y por tanto en la construcción de la situación de discapacidad. Sin restar la responsabilidad del Estado, el gestor cultural tiene la oportunidad de profesionalizar el acceso universal de las personas en situación de discapacidad a la cultura en Chile. Los desafíos giran en torno a formar agentes que tomen conciencia, promuevan y garanticen las condiciones necesarias para que disminuyan los factores contextuales ambientales que pueden generar una situación de discapacidad a potenciales audiencias, espectadores y artistas. Acciones concretas, que pueden ir desde la implementación del sistema de lecto-escritura Braille en todas las bibliotecas públicas del país hasta la adaptación estructural de una obra de teatro, todo un mundo de ideas y necesidades que queda a la creatividad de los gestores culturales.

No obstante, como el modelo de políticas públicas chileno continúa basándose en un sistema de subsidiariedad, a la hora de formular proyectos que integren la variable discapacidad, el material de documentación se hace cada vez menos accesible al momento de justificar esta urgente necesidad. Es cosa de ver cómo el próximo Censo abreviado del año 2017 excluye preguntas en torno a la discapacidad en Chile, un suministro básico para cuantificar a los potenciales espectadores o artistas que puedan aportar al bien común del país, tanto para generar políticas públicas (ya sea a través del SENADIS o del futuro Ministerio de las Culturas y las Artes) o de los mismos gestores culturales.

La creatividad no tiene límites, pero es apremiante contar con estrategias públicas que promuevan la cultura por y para todos y que sirvan como herramienta referencial para los gestores culturales motivados por garantizar el derecho básico de acceso universal a la cultura en Chile. Asimismo, es fundamental contar con mayor investigación centrada en el diagnóstico de la realidad de nuestro país a nivel cualitativo y cuantitativo.

Destacamos la intención de los programas y fondos públicos que se están ejecutando y destinando en el Consejo Nacional de la Cultura y las Artes, la Dibam y SENADIS, pero miramos con preocupación que no exista una política interministerial que se haga cargo de permitir el acceso universal a la cultura de casi tres millones de chilenos en situación de discapacidad. Si bien vemos cómo surgen nuevos proyectos con miras a la integración plena, las falencias continúan en áreas tan fundamentales como una difusión estratégica que, consideramos, va más allá de transmitir un mensaje en lengua de señas. Mucho se habla en Chile de la urgente necesidad de la formación de audiencias y nos parece indispensable que esta política cultura incluya directrices y acciones claras dirigidas a las personas en situación de discapacidad.

Las políticas públicas chilenas están gravemente en deuda con la variable de la discapacidad y lo seguirán estando si nosotros, como gestores culturales, no demandamos mayor investigación de calidad, estrategias referenciales aplicadas a las necesidades chilenas y una política que garantice recursos para hacer de la cultura el placer que absolutamente todas las personas en Chile merecen vivir a plenitud.

\section{RefERENCIAS BibliogRÁfiCAS}

Consejo Nacional de la Cultura y las Artes (2011). Política Cultural 2011-2016. Departamento de Estudios. Recuperado de: http://www.cultura.gob. cl/wp-content/uploads/2011/11/politica_cultural_2011_2016.pdf

Consejo Nacional de la Cultura y las Artes (2016). Sitio web. Sección: quienes somos. Obtenido el 01.08.16. Recuperado de: http://www.cultura.gob.cl/institucion/quienessomos/

Kay, Adrain (2006). The dymanics of public policy: Theory and evidence, United Kingdom: Edward Edgar Publishing Limited. Traducción propia.

Ministerio de Cultura y Ministerio de Sanidad, Política Social e lgualdad de España (2011). Estrategia integral española de cultura para todos. Recuperado de https://www.msssi.gob.es/ssi/discapacidad/docs/ estrategia_cultura_para_todos.pdf

Organización Mundial de la Salud (2001). Clasificación Internacional del Funcionamiento, de la Discapacidad y de la Salud. Versión abreviada. Editor Ministerio de Trabajo y Asuntos Sociales. Secretaría de Estado de Servicios Sociales, Familias y Discapacidad. Instituto de Mayores y Servicios Sociales (IMSERSO), España. Recuperado de http://apps. who.int/iris/bitstream/10665/43360/1/9241545445_spa.pdf

Organización de las Naciones Unidas (2006). Protocolo facultativo de la Convención sobre los derechos de las personas con discapacidad. Recuperado de http://www.un.org/disabilities/documents/convention/convoptprot-s.pdf

Peters, B. G. (2013). American Public Policy: Promise and Performance, United States of America: CQ Press, SAGE Publications. Novena Edición. Traducción propia. 
Servicio Nacional de la Discapacidad. (2016). Sitio Web. Descripción. Obtenido el 01.08.16 Recuperado de http://www.senadis.gob.cl/ pag/154/1220/descripcion

Servicio Nacional de la Discapacidad. (2016). Sitio web. Líneas de acción de cultura. Obtenido el 01.08.16 Recuperado de http://www.senadis. gob.cl/pag/155/1403/lineas_de_accion_de_cultura 